Cytogenet Genome Res 1997;79:303-304

\title{
Subject Index, Vol. 79, 1997
}

This index does not contain items of the chromosome $\mathrm{Y}$ and 21 mapping workshop reports. Cytogenetics and Cell Genetics

\section{Animals}

Atelespaníscus chamek (spider monkey) 92 Bos taurus (bovine) 179, 204, 225 Dicrostonyx groenlandicus (collared

lemming) 288 Feíis cattus (domestic cat) 71, 118, 145 Gobius niger, G. paganellus, G. cobitis, Zosterisessor ophiocephalus (gobiid

fishes) 266 Microtus cabrerae (vole) 167 mouse, 109,172,241,243,298 Oncorhynchus mykiss (rainbow trout) 184 Pongo pygmaeus pygmaeus (orangutan) 257 rat $109,151,172,176,235$ sheep 193 swine 79

Banded chromosome analysis bovine(GTG) 179 cat $(\mathrm{G}, \mathrm{Q}) 71$ cat $(\mathrm{Q})$ 118, 145 gobiid fishes (RBG, C, DA/DAPI, DA/

CMA3) 266 lemming(G) 288 human(DAPI) 85, 121, 123, 143, 147, 188, $198,208,210,212,221,233,237,257$,

274,276,284 human(Q) 101 human(G) 53, 103, 162 human(GTG) 228 human(R) 60, 101, 132, $139,231,257,282$,

286 mouse(G) 109 mouse(R) 109, 172,241 $\operatorname{rat}(\mathrm{R})$ 109, 151, 172,241 $\operatorname{rat}(\mathrm{G}) 109$

orangutan(DAPI) 257 swine(R) 79 sheep(DAPI) 193

Cell cycle

chromosome stretchability 162 Chromosome aberration

amplified region 125

deletions 85,276,293

fragile site 88

paracentric inversion 257

rearrangements 257, 266, 293

translocations 153 Chromosome mapping workshops

chromosome 2121

Y chromosome 1 Chromosome stretching

GTG band analysis 162 Chromosome transfer

selectable subchromosomal fragments 53 Comparative gene mapping

bovine $179,204,225$

cat 118

humans 214

mouse $172,179,298$

orangutan 257

rat $149,151,172,235$

sheep 193 spider monkey 92 Consensus map

chromosome 10257 


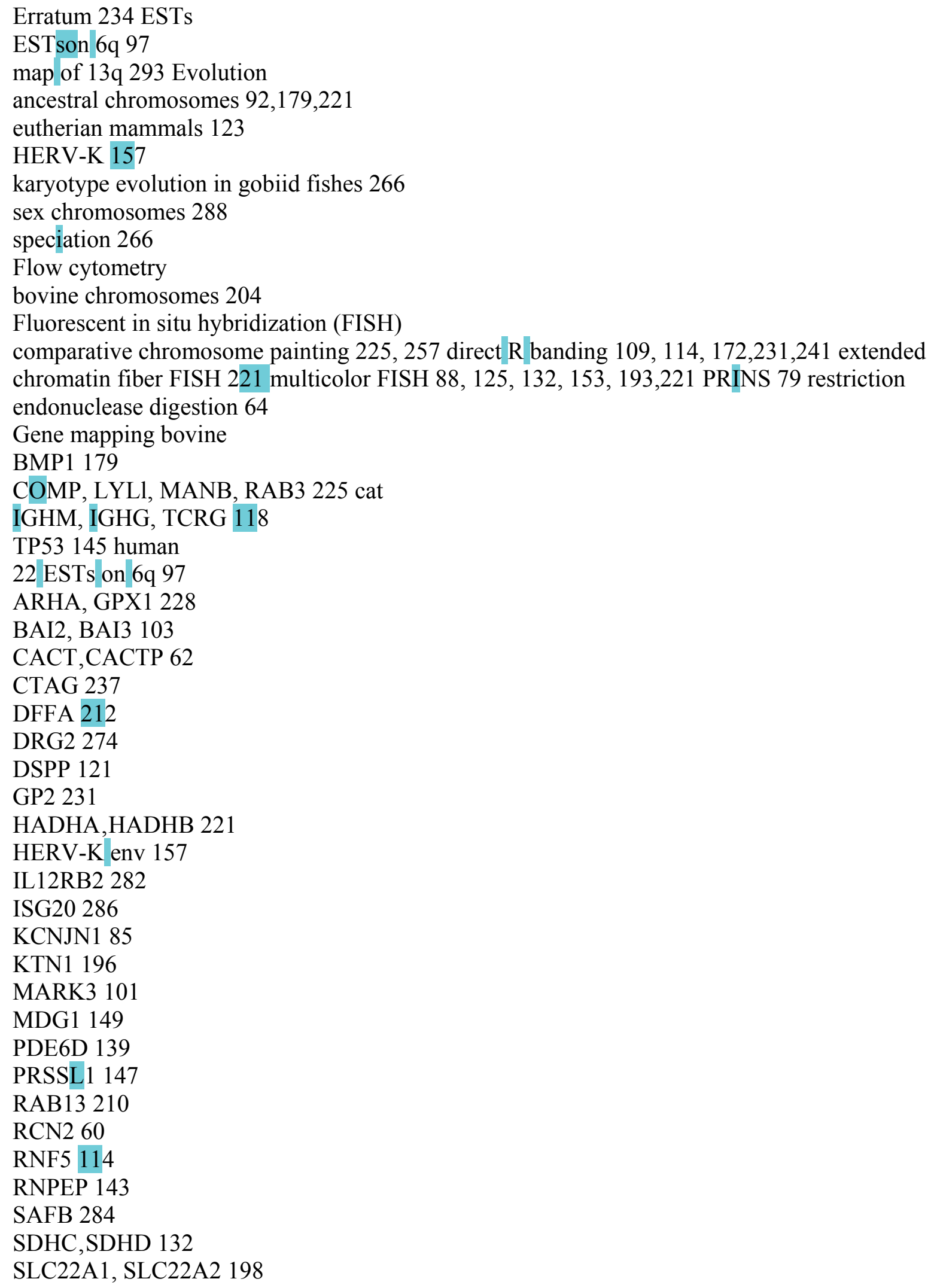


SSP2 142

STAT6 208

STK6, STK6P 201

TNNI3 272

TRO 123

tumor suppressor gene 53,97, 147

UBE2L4 188

UGCG 233 monkey

B2M, CALM1, CKB, FES, TCF1, TGM1,THBS1 92 mouse

Cenpa 298

Dgcró 243

Nat4 172

Pldl, Pld2 109

Umod 241 rat

Cdld 235

Cp 176

Nat4 172

Pc 151

Pldl, Pld2 109

Umod 241 sheep

TCRA, TCRD 193 Genetic damage

fragile site induction 88 gene amplification 125 ionizing radiation 153 Gene organization CTAG 237 RNF5 114

Heterochromatin

location in gobiid fish species 266 Human disorders

aceruloplasminemia 176

Bardet-Biedl syndrome 60

breastcancer $125,201,248,284$

carcinomas 237

dentinogenesis imperfecta type II 121

DiGeorge syndrome 243

germ cell tumors 157

glioblastoma 103

hypertrophic cardiomyopathy 272

melanoma 53

mental retardation 276, 293

mitochondrial dysfunction 132

neurological disorders 293

neuronal ceroid lipofuscinosis 293

neuropsychiatric disorders 214

nocturnal enuresis type 1293

oncogenic virus infection 286

Rieger syndrome 293

Smith-Magenis syndrome 85,276

thyroid cancer 153

tumorigenesis 114 Hybrids 
ateles-rodent 92

bovine-rodent 179, 204, 225

breakpoint region 97

chromosome 6 hybrid panel 97

human-rodent panel 53, 97, 157, 237, 276, 282,284,298

\section{KARGEH}

Fax + 41613061234 E-Mail karger@,karger.ch www.karger.com

(C) 1998 S.KargerAG, Basel

This article is also accessible online at: http://BioMedNet.com/karger

303

microcell hybrids chromosome 1153 radiation hybrid panel 53, 114, 147,257,

282 somatic cell hybrid panel 62, 188, 198,201,

228

In situ hybridization ISG20 286

Karyotype

cat 71

collared lemming 288

Linkage

analysis by 2-D DNA typing 248 bovine chromosomes 179, 204 chromosome 10 map 257

interspecific backcross analysis 172,176 ,

298 rat chromosome 2 map 176

Meiosis

neo-XY sex chromosomes 288

Physical mapping

$1 \mathrm{~kb}$ resolution map $64 \mathrm{llq}$ amplified region 125 bovine chromosome 204, 225 chromosome 10 257 ESTs on $6 q 97$

Repeats

anxiety-related traits 214

regulatory properties 214

screening by 2-D DNA typing 248

swine centromeric repeats 79

trout chromosome specific markers 184

Restriction mapping APOB 64

Sequence BAIs 103 HERV-Kéw 157 MARK3 101 mouse Dgcró 243 PDE6D 139 ratPLD 109

RNF5 114

SDHC,SDHD 132

SLC6A4 214

SRY(vole) 167

STK6, STK6P 201

trout chromosome-specific marker 184

UBE2L4 188

Sex chromosomes

localization of CT antigens 237 multiple copies of SRY 167 neo-XY derivatives in lemmings 288

Techniques 
2-D DNA typing 248 isolation of alternate transcripts 188 PTT protein truncation test 157 QDFM for high resolution mapping 64

304

Cytogenet Cell Genet Vol. 79, 1997 